\title{
Forming Mechanism and Correction of CT Image Artifacts Caused by the Errors of Three System Parameters
}

\author{
Ming Chen and Gang Li \\ College of Information Science and Engineering, Shandong University of Science and Technology, Qingdao 266590, China \\ Correspondence should be addressed to Gang Li; ligangccm@163.com
}

Received 27 January 2013; Accepted 30 March 2013

Academic Editor: Hang Joon Jo

Copyright ( 2013 M. Chen and G. Li. This is an open access article distributed under the Creative Commons Attribution License, which permits unrestricted use, distribution, and reproduction in any medium, provided the original work is properly cited.

\begin{abstract}
We know that three system parameters, a center of X-ray source, an isocenter, and a center of linear detectors, are very difficult to be calibrated in industrial CT system. So there are often the offset of an isocenter and the deflection of linear detectors. When still using the FBP (filtered backprojection) algorithm under this condition, CT image artifacts will happen and then can seriously affect test results. In this paper, we give the appearances and forming mechanism of these artifacts and propose the reconstruction algorithm including a deflection angle of linear detectors. The numerical experiments with simulated data have validated that our propose algorithm can correct CT images artifacts without data rebinning.
\end{abstract}

\section{Introduction}

We usually adopt the FBP (filtered backprojection) algorithm in industrial CT. This algorithm requires two necessary conditions [1,2]: (i) the isoray (an imaginary ray that connects a center of X-ray source with an isocenter) is perpendicular to linear detectors; (ii) an insection point where the isoray and linear detectors cross is the center of linear detectors. However, a center of X-ray source, an isocenter, and a center of linear detectors are difficult to be calibrated in industrial CT. When still using the FBP algorithm under the errors of three system parameters, the image artifacts will happen and then can seriously affect test results, especially for these reconstructed points away from the center of CT images.

When there happens the offset of an isocenter, people usually take the projection point of the isocenter on linear detectors as the center of linear detectors and then translate the projections. For parallel beam, this translation can recalibrate the isocenter [3]. However for fan beam, this translation is impossible unless fan beam projections are rebinned as parallel beam projections [4]. For measuring and correcting the CT system parameters, there are some methods proposed. Gullberg et al. [5] proposed the method to correct the isocenter for fan beam; however, the involved parameters are difficult to obtain. Sun et al. [6] assumed that the plane of four small balls is perpendicular to the turn table and then measured cone-beam CT system parameters by use of projection data under one angle. For micro-CT, Patel et al. [7] proposed the autocalibration method without model measurement and measured and corrected some system parameters. For conebeam CT, Chen et al. [8] estimated some parameters by obtaining the barycenter under the condition that the plane detector is parallel to the axis of rotation.

The remainder of this paper is organized as follows. In Section 2, we introduce the FBP algorithm for fan beam and point out its necessary conditions. In Section 3, we give the appearances of three image artifacts caused by the offset of an isocenter and the deflection of linear detectors. In Section 4, we analyze the forming mechanism of three artifacts. In Section 5, we propose the FBP algorithm including a deflection angle of linear detectors. Finally, numerical experiments and conclusions are presented in Section 6.

\section{The FBP Algorithm for Fan Beam}

For convenience of the formula derivation in Section 5, we introduce the FBP algorithm for equal-spaced fan beam in this section. 


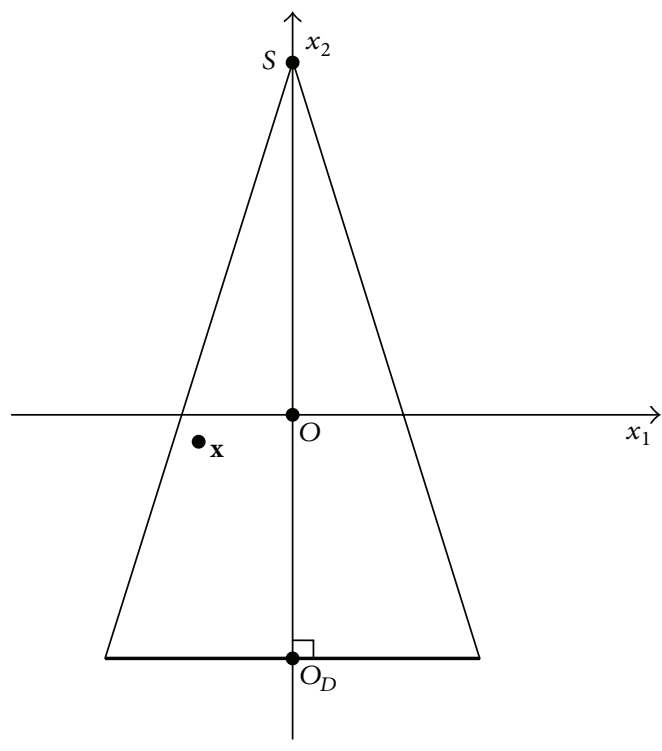

FIGURE 1: A simple geometric relationship of an equal-spaced fan beam.

A simple geometric relationship with no errors of CT system parameters is shown in Figure 1. We define a righthanded coordinate system $O x_{1} x_{2}$, where the origin $O$ is an isocenter, $x_{1}$ axis is parallel to linear detectors (the bold line in Figure 1), and $x_{2}$ axis is parallel to the isoray. Let $R_{1}$ denote the distance from $\mathrm{X}$-ray source to $x_{1}$ axis (if the isoray is perpendicular to linear detectors, $R_{1}$ is also the distance between $\mathrm{X}$-ray source $S$ and the isocenter $O$ ), and let $R_{2}$ denote the distance between $S$ and the center $O_{D}$ of linear detectors. Let $p(\beta, s)$ denote the equal-spaced fan beam projection data, where $\beta$ is the angle of the isoray formed with the $x_{2}$ axis and $s$ is a sample on the imaginary detectors which are through the isocenter $O$ and parallel to the actual linear detectors. Making use of the FBP reconstruction algorithm, the image function, $f(\mathbf{x})=f\left(x_{1}, x_{2}\right)$, can be shown to be

$$
\begin{aligned}
f(\mathbf{x})= & \int_{0}^{2 \pi} \frac{R_{1}{ }^{2}}{\left(R_{1}-\mathbf{x} \cdot \beta^{\perp}\right)^{2}} \\
& \times\left.\left\{\frac{R_{1}}{\sqrt{R_{1}{ }^{2}+s^{2}}} p(\beta, s) * h(s)\right\}\right|_{s=s_{0}} d \beta,
\end{aligned}
$$

where $s_{0}$ is the projection address of a reconstructed point $\mathbf{x}$ on the imaginary detectors, $\beta=(\cos \beta, \sin \beta), \beta^{\perp}=$ $(-\sin \beta, \cos \beta), s_{0}=\left(R_{1} \mathbf{x} \cdot \beta\right) /\left(R_{1}-\mathbf{x} \cdot \beta^{\perp}\right)$, and $h(s)=$ $\int_{-\infty}^{+\infty}|\omega| e^{i 2 \pi \omega s} d \omega$ is a filter function.

According to a geometric relationship in Figure 1, the FBP algorithm requires two necessary conditions: (i) the isoray is perpendicular to linear detectors; (ii) an insection point where the isoray and linear detectors cross is the center of linear detectors.

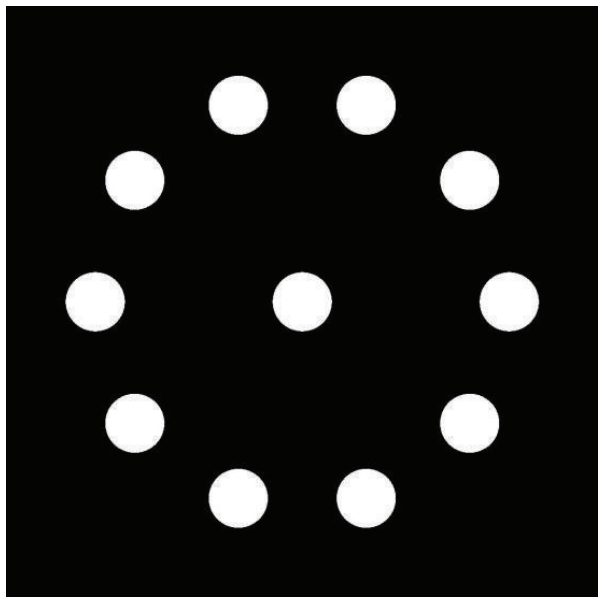

Figure 2: Phantom.

\section{Appearances of Three Image Artifacts}

We give a test phantom, which is comprised of eleven circles, and ten circles are distributed evenly among the center of the phantom, as shown in Figure 2.

The offset of an isocenter may cause CT image artifacts [9-12]. This offset is divided into two cases: along linear detectors or along the direction which is perpendicular to linear detectors. The latter is equivalent to error of $R_{1}$. When $R_{1}$ is much larger than the field of view (FOV), we can still reconstruct a satisfying CT image, even if $R_{1}$ remains some errors [13]. For this reason, we only consider the offset of an isocenter along linear detectors.

We give a simple geometric relationship of CT scanning system with the offset of an isocenter in Figure 3, where $O_{1}$ is the isocenter. Let $x_{2}$ axis denote the direction which is through the center $O_{D}$ of linear detectors and perpendicular to linear detectors. Let $x_{1}$ axis denote the direction which is through $O_{1}$ and perpendicular to $x_{2}$ axis. Let $O$ denote the insection point where the $x_{1}$ axis and the $x_{2}$ axis cross. Let $\gamma$ denote the angle contained by the line $O S$ and the line $O_{1} S$.

We perform numerical experiments with the simulated data to show the appearance of image artifacts caused by the offset of an isocenter. CT scanning system parameters are as follows: the distance from $\mathrm{X}$-ray source $S$ to $x_{1}$ axis $R_{1}=550.000 \mathrm{~mm}$, the distance from X-ray source $S$ to linear detectors $R_{2}=905.000 \mathrm{~mm}$, linear detectors are composed of 1024 cells, with the size of each cell $0.4 \mathrm{~mm}$. We assume that there is the offset of an isocenter $\left|O O_{1}\right|=5.0 \mathrm{~mm}$. Each detector takes 720 projections in $2 \pi$. The image matrix is $1024 \times 1024$. For the phantom in Figure 2, we reconstruct CT images using the FBP formula (1), as shown in Figure 4, where the artifacts nonuniformly spread to all directions. And the reconstructed points away from the center of CT images are comparatively worse.

For this offset of an isocenter, we may obtain the projection point of the isocenter on linear detectors by many experiments and then translate the projection data. The reconstructed images from the translated projection data are 


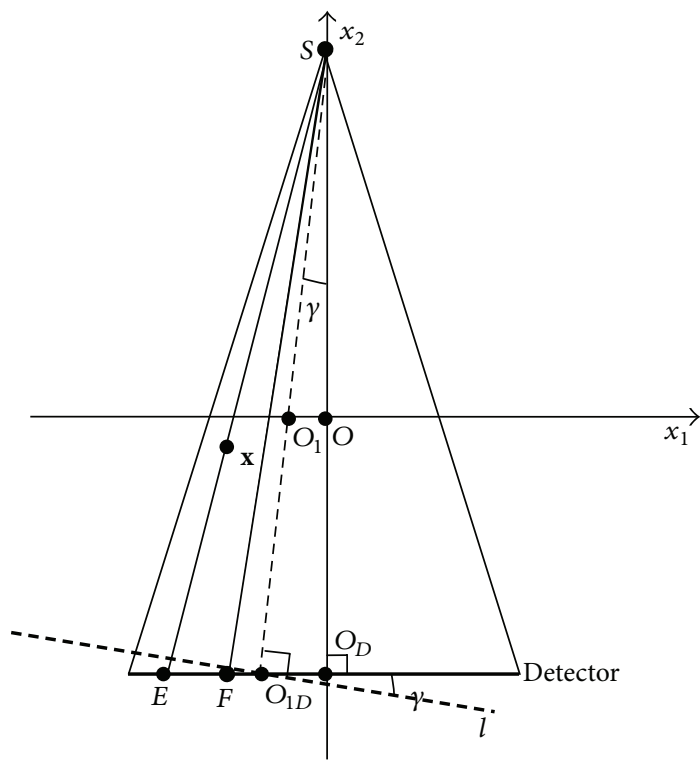

FIGURE 3: A simple geometric relationship of CT scanning system with the offset of an isocenter.
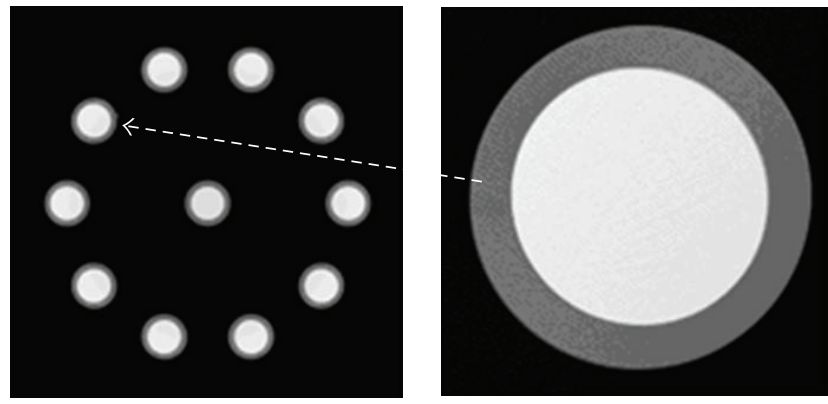

FIGURE 4: CT image artifacts caused by the offset of an isocenter.

shown in Figure 5, where the artifacts obviously reduce. In fact, an isoray is not perpendicular to linear detectors when the offset of an isocenter happens. So there still exist some image artifacts caused by the deflection of linear detectors in Figure 5. That is, linear detectors deflect to the dotted line from $x_{1}$ axis in Figure 3.

We also give a simple geometric relationship of CT scanning system with the deflection of linear detectors in Figure 6, where linear detectors deflect to the heavy continuous line from $x_{1}$ axis. Let $\gamma$ denote the clockwise deflection angle. Making use of the same previous parameters, we can calculate $\gamma=0.52^{\circ}$. We can reconstruct CT images using the FBP formula (1) from the projection data with the deflection of linear detectors, as shown in Figure 5, which is exactly same with the reconstructed image from the translated projections data with the offset of isocenter.

Similarly, when the offset of an isocenter and the deflection of linear detectors simultaneously happen, we also obtain CT image artifacts, as shown in Figure 7, where the isocenter offset is $2.0 \mathrm{~mm}$ and $\gamma=0.52^{\circ}$, and the other parameters are same as above mentioned.
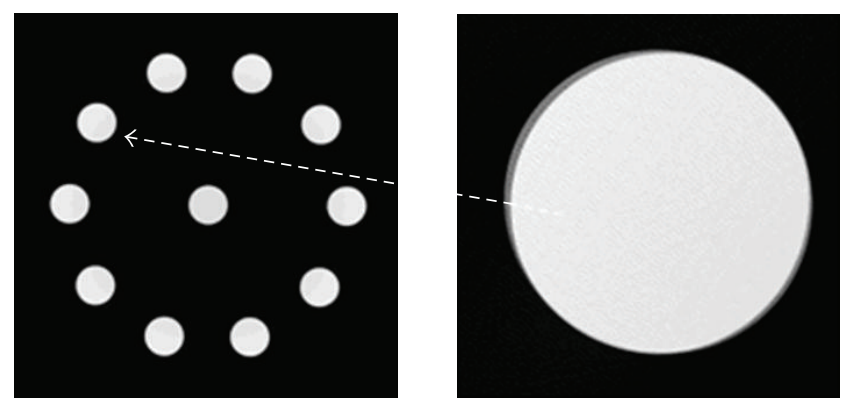

FIgURE 5: Reconstruction images from the translated projection data, or CT image artifact caused by the deflection of linear detectors.

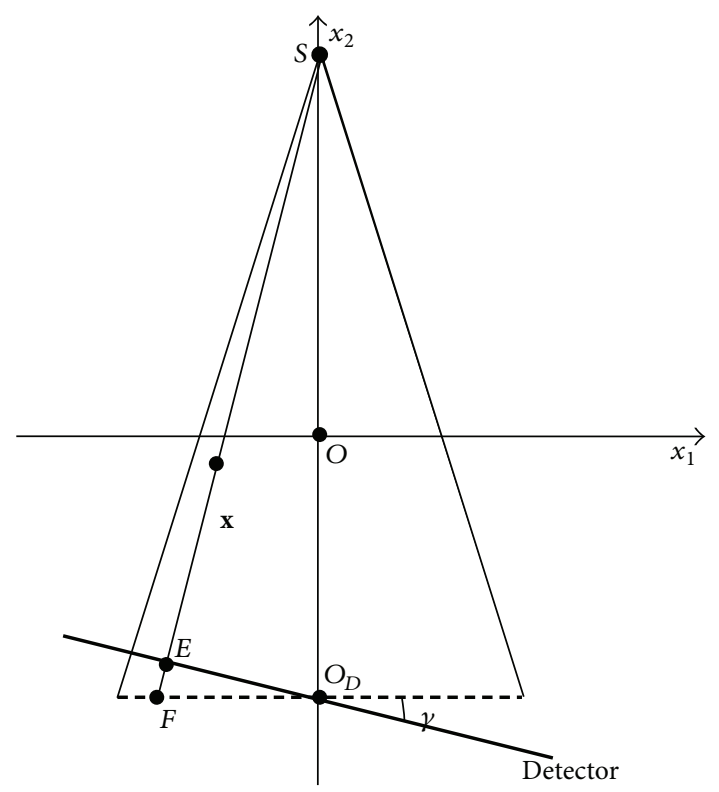

FIGURE 6: A simple geometric relationship of CT scanning system with the deflection of linear detectors.

\section{Forming Mechanism of Three Image Artifacts}

We give the forming mechanism of three image artifacts in this section. For a reconstructed point $\mathbf{x}$, we analyze a reconstruction process of $\mathbf{x}$ and give a minimum bias expression under every projection angle.

For ease of the following analysis, let a polar coordinate $(r, \theta)$ denote $\mathbf{x}$, and let $s_{0}$ denote its projection address on linear detectors. If there is no error in CT system, we can calculate $s_{0}=R_{2} \times r \cos (\beta-\theta) /\left(R_{1}+r \sin (\beta-\theta)\right)$. From Figure 3 , the projection point of $\mathbf{x}$ is a point $E$ on linear detectors, and a projection address is $s=O_{1 D} E$, where $O_{1 D}$ is the projection point of the isocenter $O_{1}$ on linear detectors. However, we still take $O$ as an isocenter in image reconstruction when using the FBP formula (1). So the other point $F$ is regarded as the projection point of $\mathbf{x}$ where $O_{D} F=$ $O_{1 D} E$. Under this condition, $\mathbf{x}$ will be reconstructed on the line $S F$. Now, we draw a vertical line which is through $\mathbf{x}$ 

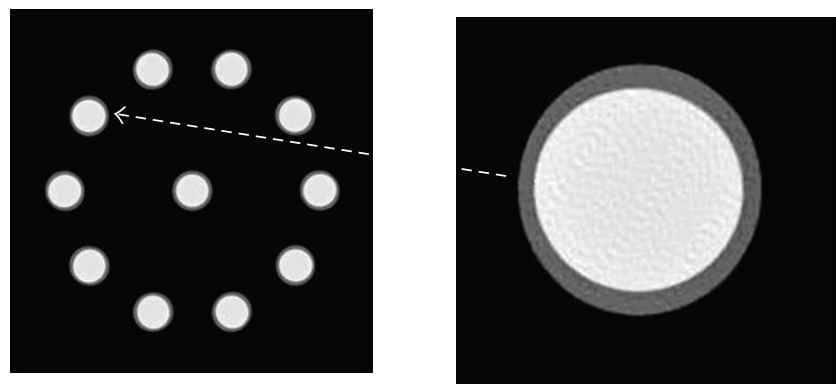

FIGURE 7: CT image artifacts caused by the offset of an isocenter and the deflection of linear detectors.

and perpendicular to the line $S F$, and let $M(\beta)$ denote the insection point. The trajectory of $M(\beta)$ can approximately describe the reconstruction result of $\mathbf{x}$ when $\beta$ ranges from 0 to $2 \pi$. Now, firstly we calculate the distance $R(\beta)$ between $\mathbf{x}$ and $M(\beta)$ as follows:

$$
\begin{aligned}
R(\beta)= & \left.|| O S\right|^{2} \times r \cos (\beta-\theta)-s \times\left|O_{D} S\right| \times|O S| \\
& -s \times\left|O_{D} S\right| \times r \sin (\beta-\theta) \mid \\
& \times\left(\sqrt{s^{2} \times\left|O_{D} S\right|^{2}+|O S|^{4}}\right)^{-1},
\end{aligned}
$$

where $s=s_{0}-\left(\left|O_{D} S\right| \times\left|O O_{1}\right| /|O S|\right)$.

So, we can obtain a coordinate of $M(\beta)$ as follows:

$$
\begin{aligned}
M(\beta)= & \left(r \cos \theta-R(\beta) \times \cos \left(\beta+\tan ^{-1} \frac{s \times\left|O_{D} S\right|}{|O S|^{2}}\right),\right. \\
& \left.r \sin \theta-R(\beta) \times \sin \left(\beta+\tan ^{-1} \frac{s \times\left|O_{D} S\right|}{|O S|^{2}}\right)\right) .
\end{aligned}
$$

We choose a reconstructed point $\mathbf{x}_{0}=(90, \pi / 4)$ and assume that the offset $\left|O O_{1}\right|$ of an isocenter is $0.744 \mathrm{~mm}$, that is, 2.15 pixel. According to formula (3), we may draw the trajectory of $M(\beta)$ by Mathematica, where $\beta$ ranges from 0 to $2 \pi$, as shown in Figure 8(a). The reconstruction image of $\mathbf{x}_{0}$ using the FBP formula (1) is shown in Figure 8(b), which explain the artifacts in Figure 4.

Similarly, for the linear detectors deflection, we may calculate the same previous expressions (2) and (3) of $R(\beta)$ and $M(\beta)$, where $s=s_{0} \sin \alpha / \sin (\gamma+\alpha), \alpha=\tan ^{-1}\left(s_{0} /|O S|\right)$. We choose $\gamma=0.52^{\circ}$. The trajectory of $M(\beta)$ and the reconstruction image of $\mathbf{x}_{0}$ are as shown in Figure 9, which explain the artifacts in Figure 5.

Similarly, for the offset of an isocenter and the deflection of linear detectors, we also calculate the previous expressions (2) and (3) of $R(\beta)$ and $M(\beta)$, where $s=\left(s_{0} \times|O S|-\left|O_{D} S\right| \times\right.$ $\left.\left|O O_{1}\right|\right) \times \sin \alpha /|O S| \times \sin (\gamma+\alpha), \alpha=\tan ^{-1}\left(s_{0} /|O S|\right)$. We choose the offset of an isocenter $\left|O O_{1}\right|=0.5 \mathrm{~mm}$ and $\gamma=$ $0.6^{\circ}$. The trajectory of $M(\beta)$ and the reconstruction image of $\mathbf{x}_{0}$ are as shown in Figure 10, which explain the artifacts in Figure 7.

\section{Derivation of FBP Formula Including a Deflection Angle of Linear Detectors}

In this section, we describe a new coordinate system and derive the FBP formula including a deflection angle of linear detectors, where the offset of an isocenter is attributed to the deflection of linear detector.

Referring to Figure 11, we establish the coordinate system $O x_{1} x_{2}$, where the origin $O$ is the isocenter, $x_{2}$ axis is parallel to the isoray and points to $\mathrm{X}$-ray source $S$, and $x_{1}$ axis and $x_{2}$ axis form right-handed coordinate system. Let $\varphi$ denote the angle contained by the $x_{1}$ axis and linear detectors. Obviously, $x_{2}$ axis is not perpendicular to linear detectors, and there is a deflection of linear detectors and no offset of an isocenter in this system.

For convenience of derivation, let the polar coordinate $f(r, \theta)$ denote the image function. Let $O^{\prime}$ denote the projection point of the isocenter $O$ on linear detectors, $R_{1}=|O S|$ and $R_{2}=\left|O^{\prime} S\right|$. We use the imaginary detectors in formula derivation. Let $d, q$, and $s$ denote three projection points of the reconstructed point $\mathbf{x}=(r, \theta)$ on linear detectors, the imaginary detectors, and $x_{1}$ axis, respectively. Let $p(d, \beta)$, $p_{1}(q, \beta)$, and $p_{2}(s, \beta)$ denote the corresponding projection data. For a reconstructed point $\mathbf{x}_{0}=\left(r_{0}, \theta_{0}\right)$, and let $d_{0}, q_{0}$, and $s_{0}$ denote three projection points corresponding to $\mathbf{x}_{0}$, respectively.

From Figure 11, we can obtain the relationship between $s_{0}$ and $q_{0}, s$, and $q$ as follows:

$$
\begin{gathered}
s_{0}=\frac{R_{1} q_{0} \cos \varphi}{R_{1}-q_{0} \sin \varphi}, \\
s=\frac{R_{1} q \cos \varphi}{R_{1}-q \sin \varphi} .
\end{gathered}
$$

Now, we rewrite the FBP formula (1) as follows:

$$
\begin{aligned}
f\left(r_{0}, \theta_{0}\right)= & \frac{1}{2} \int_{0}^{2 \pi} \frac{R_{1}{ }^{2}}{\left(R_{1}-r_{0} \sin \left(\theta_{0}-\beta\right)\right)^{2}} \\
& \times \int_{-\infty}^{\infty} \frac{R_{1}}{\sqrt{R_{1}^{2}+s^{2}}} p_{2}(s, \beta) h\left(s_{0}-s\right) d s d \beta,
\end{aligned}
$$

where $h(s)=\int_{-\infty}^{\infty}|\omega| e^{i 2 \pi \omega s} d \omega, s_{0}=R_{1} r_{0} \cos \left(\theta_{0}-\beta\right) /\left(R_{1}-\right.$ $\left.r_{0} \sin \left(\theta_{0}-\beta\right)\right)$.

From formula (4), (5), and (6), we may obtain

$$
q_{0}=\frac{R_{1} r_{0} \cos \left(\theta_{0}-\beta\right)}{R_{1} \cos \varphi-r_{0} \sin \left(\theta_{0}-\beta-\varphi\right)} .
$$

From formula (5) and $h(s)$, we can obtain

$$
\begin{gathered}
\frac{d s}{d q}=\frac{R_{1}{ }^{2} \cos \varphi}{\left(R_{1}-q \sin \varphi\right)^{2}}, \\
h\left(s_{0}-s\right)=\frac{1}{C^{2}} h\left(q_{0}-q\right),
\end{gathered}
$$

where $C=R_{1}^{2} \cos \varphi /\left(R_{1}-q_{0} \sin \varphi\right)\left(R_{1}-q \sin \varphi\right)$. 


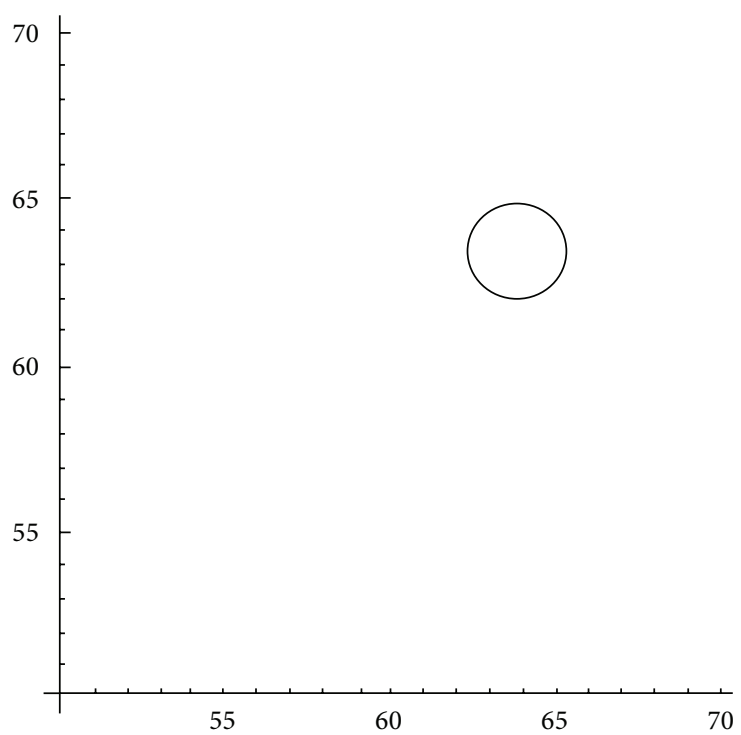

(a)

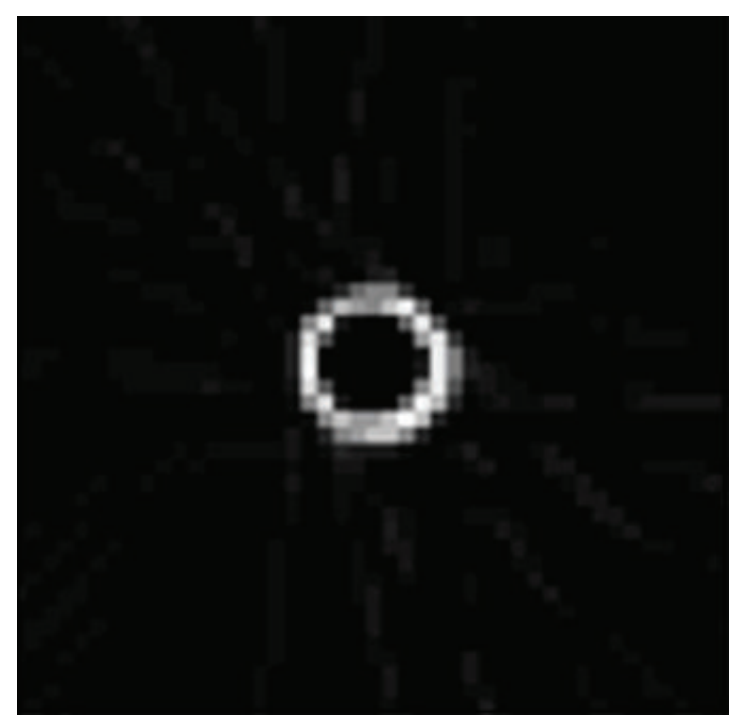

(b)

Figure 8: Analysis of artifacts caused by the offset of an isocenter: (a) the trajectory of $M(\beta)$; (b) the reconstruction image.

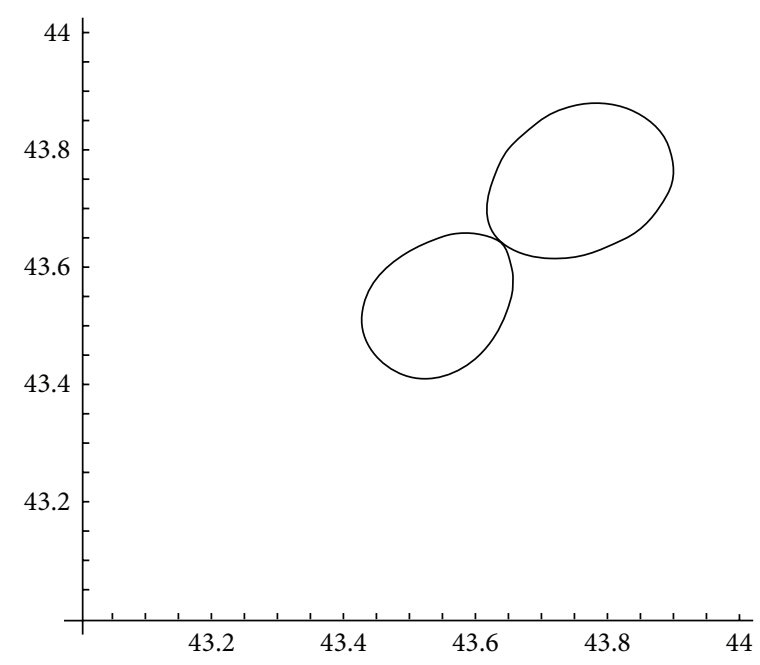

(a)

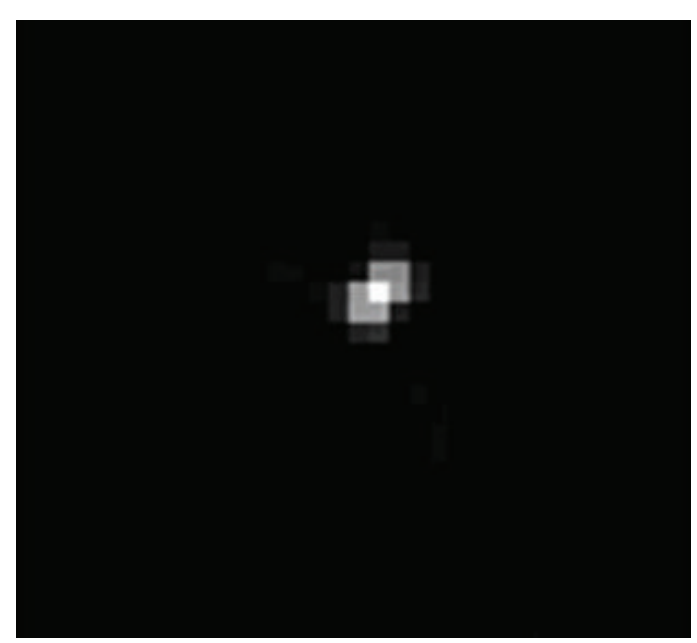

(b)

FIGURE 9: Analysis of artifacts caused by the deflection of linear detectors: (a) the trajectory of $M(\beta)$; (b) the reconstruction image.

Finally, we substitute formulae (5) and (8) into (6) and obtain after simplifying

$$
\begin{aligned}
f\left(r_{0}, \theta_{0}\right)= & \frac{1}{2} \int_{0}^{2 \pi} \frac{\left(R_{1}-q_{0} \sin \varphi\right)^{2}}{\left(R_{1}-r_{0} \sin \left(\theta_{0}-\beta\right)\right)^{2} \cos \varphi} \\
& \times \int_{-\infty}^{\infty} \frac{R_{1}-q \sin \varphi}{\sqrt{R_{1}^{2}+q^{2}-2 R_{1} q \sin \varphi}} \\
& \times p_{1}(q, \beta) h\left(q_{0}-q\right) d q d \beta,
\end{aligned}
$$

where $p_{1}(q, \beta)=p\left(R_{2} q / R_{1}, \beta\right)$.
The proposed previous formula can directly reconstruct CT image without data rebinning. The formula includes three parameters $R_{1}, R_{2}$, and $\varphi$, which are unknown, independence from the inspected objects, and identified by CT system. For obtaining three parameters, we have designed the model with a dense matter such as iron or steel, by a row of mutual parallel width and of the slit spacing formed. By super precise scanning for the model in $2 \pi$, we could make use of the geometric relationship of these slit spacing projection and estimate three parameters. But, this method is very sensitive to a deflection angle of linear detectors $\varphi$. We can improve measurement precision by averaging the testing values of repeated measurements. 


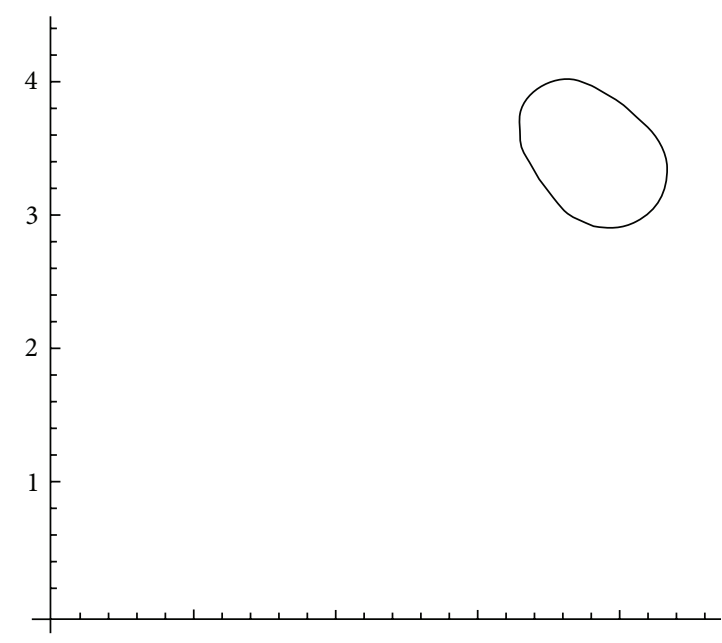

(a)

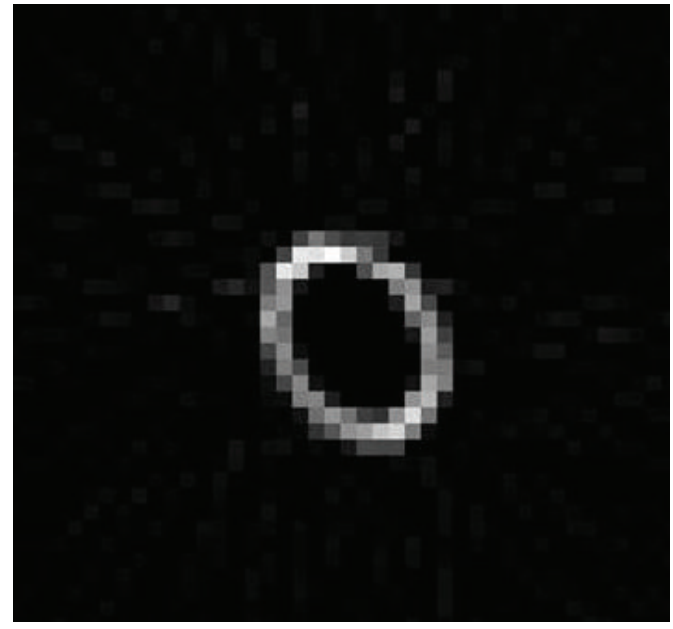

(b)

Figure 10: Analysis of artifacts caused by the offset of an isocenter and the deflection of linear detectors: (a) the trajectory of $M(\beta)$; (b) the reconstruction image.

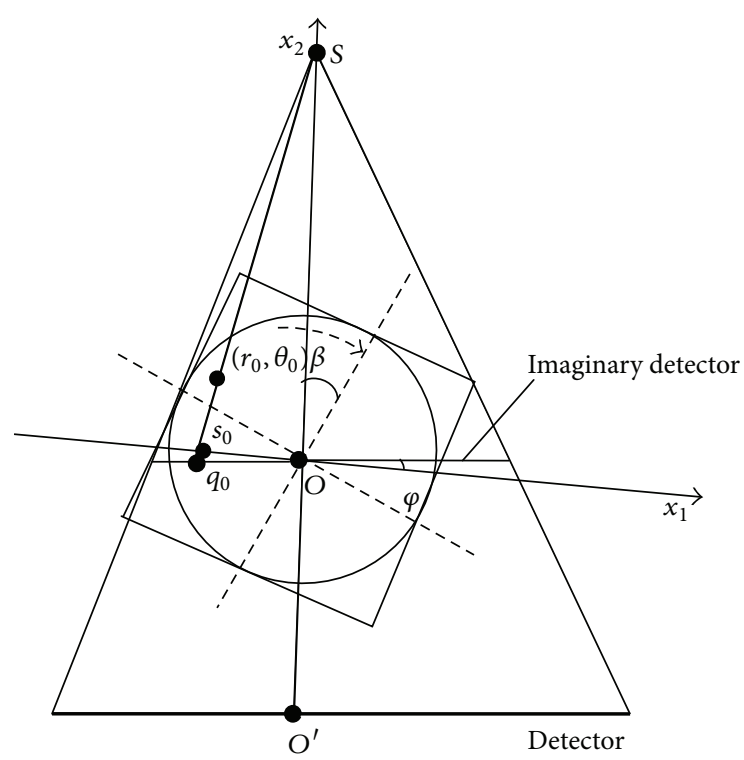

FIGURE 11: A geometric relationship of FBP formula derivation including a deflection angle of linear detectors.

\section{Numerical Simulation Experiment and Conclusion}

In this section we perform numerical experiments with simulated data to demonstrate our formula (9). We choose the phantom in Figure 2 and the system parameters in Figure 4. We can estimate $R_{1}=550.023 \mathrm{~mm}, R_{2}=905.014 \mathrm{~mm}$, and $\varphi=0.52^{\circ}$ in the formula (9). The reconstruction results are shown in Figure 12 using the formula (9). Obviously, the results validate our formula, which can correct the image
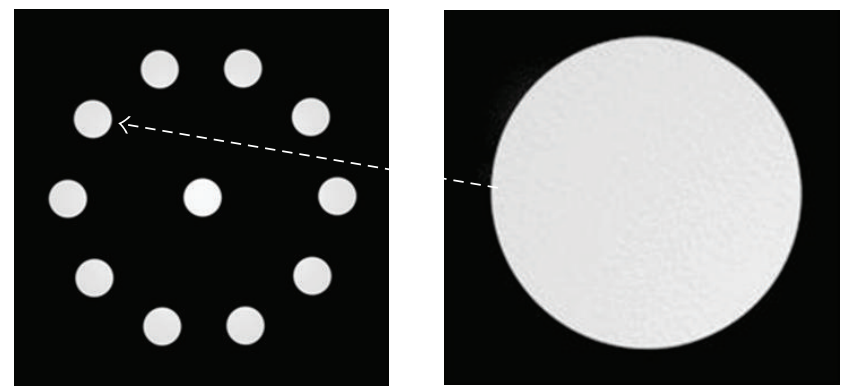

FIGURE 12: Reconstruction images using the FBP formula (9) including a deflection angle of linear detectors.

artifacts caused by the offset of an isocenter and the deflection of linear detectors.

We have given the appearances of three image artifacts caused by the offset of an isocenter and the deflection of linear detectors and analyzed the forming mechanism, which can provide reference for three artifacts identification. The correction method of the image artifacts is also proposed. Our FBP algorithm including a deflection angle of linear detectors can effectively correct three artifacts in CT images.

\section{Acknowledgments}

This work was supported in part by three Grants from the National Natural Science Foundation of China (61201430, 61002041, and 61201431), International Scientific and Technological Cooperation Program of Shenzhen (Grant JC201105190923A), China Postdoctoral Science Foundation and Shandong Province Postdoctoral Innovation Foundation. 


\section{References}

[1] A. C. Kak and M. Slaney, Principles of Computerized Tomographic Imaging, IEEE Press, New York, NY, USA, 1988.

[2] B. K. P. Horn, "Fan-beam reconstruction methods," Proceedings of the IEEE, vol. 67, no. 12, pp. 1616-1623, 1979.

[3] M. Dennis, R. Waggener, W. McDavid, W. Payne, and V. Sank, "Processing X-ray transmission data in CT scanning," Optical Engineering, vol. 16, no. 2, pp. 6-10, 1977.

[4] P. Dreike and D. P. Boyd, "Convolution reconstruction of fan beam projections," Computer Graphics and Image Processing, vol. 5, no. 4, pp. 459-469, 1976.

[5] G. T. Gullberg, C. R. Crawford, and B. M. W. Tsui, "Reconstruction algorithm for fan beam with a displaced center-ofrotation," IEEE Transactions on Medical Imaging, vol. MI-5, no. 1, pp. 23-29, 1986.

[6] Y. Sun, Y. Hou, and J. Hu, "Reduction of artifacts induced by misaligned geometry in cone-beam CT," IEEE Transactions on Biomedical Engineering, vol. 54, no. 8, pp. 1461-1471, 2007.

[7] V. Patel, R. N. Chityala, K. R. Hoffmann et al., "Self- calibration of a cone- beam micro-CT system," Medical Physics, vol. 36, no. 1, pp. 48-58, 2009.

[8] L. Chen, Z. Wu, X. Liu, and M. Yao, "Analytical geometric parameter calibration algorithm for cone-beam CT," Journal of Tsinghua University, vol. 50, no. 3, pp. 418-421, 2010.

[9] J. Li, R. J. Jaszczak, K. L. Greer, and R. E. Coleman, “A filtered backprojection algorithm for pinhole SPECT with a displaced centre of rotation," Physics in Medicine and Biology, vol. 39, no. 1, pp. 165-176, 1994.

[10] J. Li, R. J. Jaszczak, H. Wang, G. T. Gullberg, K. L. Greer, and R. E. Coleman, "A cone beam SPECT reconstruction algorithm with a displaced center of rotation," Medical Physics, vol. 21, no. 1, pp. 145-152, 1994.

[11] H. Wang, M. F. Smith, C. D. Stone, and R. J. Jaszczak, "Astigmatic single photon emission computed tomography imaging with a displaced center of rotation," Medical Physics, vol. 25, no. 8, pp. 1493-1501, 1998.

[12] Z. B. Wang, "Effect of center deviation on CT reconstruction images," Acta Armamentarii, vol. 22, no. 3, pp. 323-326, 2001.

[13] H. N. Lu, M. Yang, and L. Zhang, "A study on the reconstruction bias originating from error of focal distance of x-ray source," Acta Armamentarii, vol. 24, no. 1, pp. 65-67, 2003. 


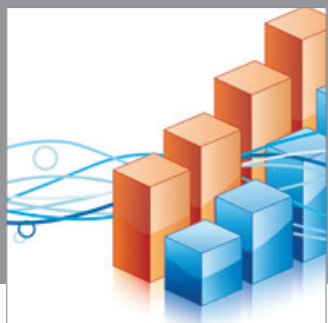

Advances in

Operations Research

mansans

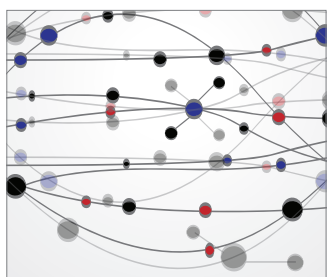

The Scientific World Journal
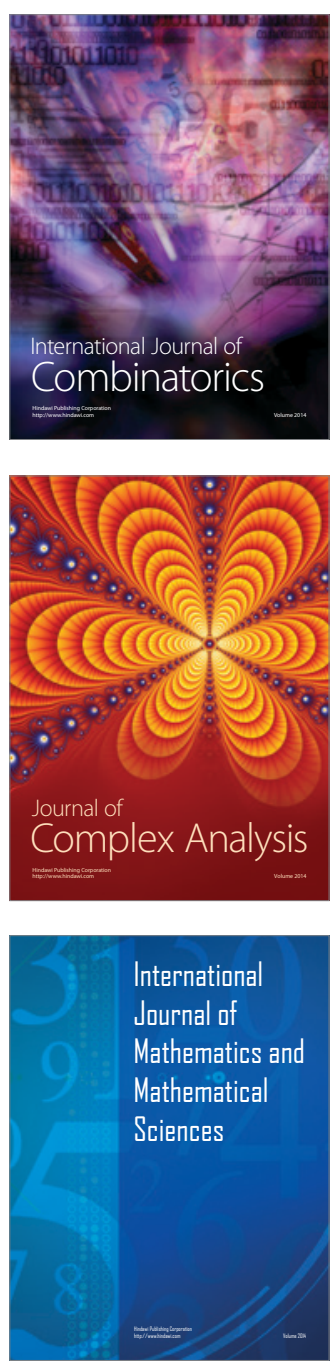
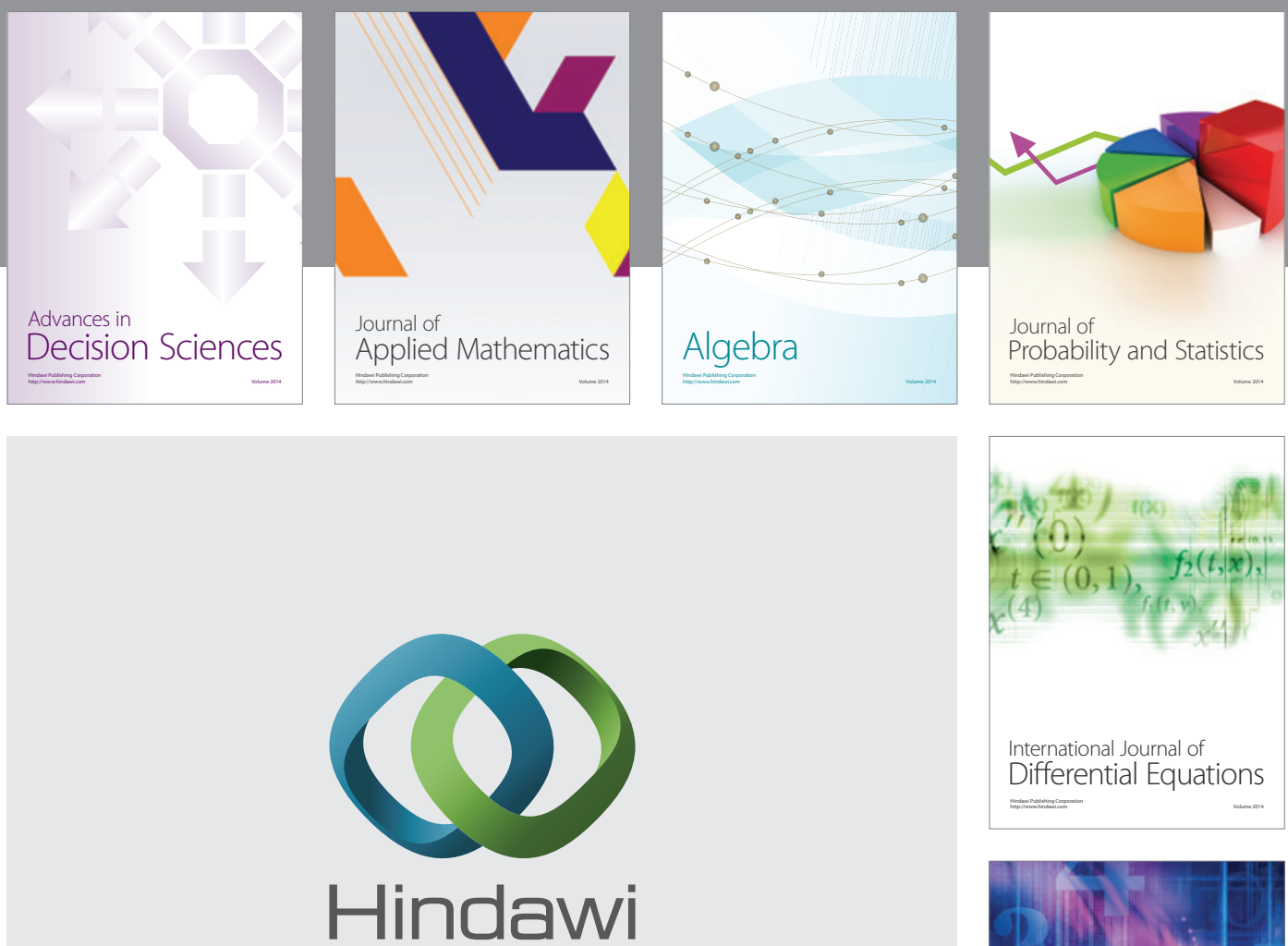

Submit your manuscripts at http://www.hindawi.com
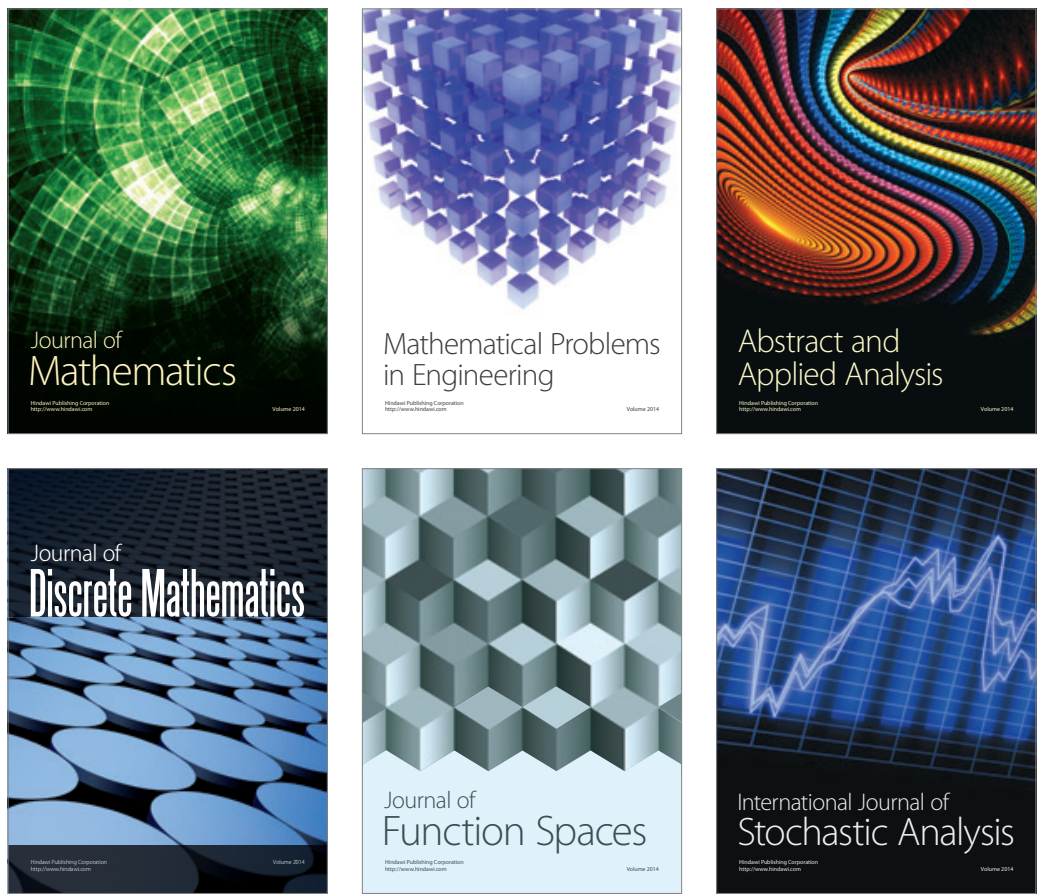

Journal of

Function Spaces

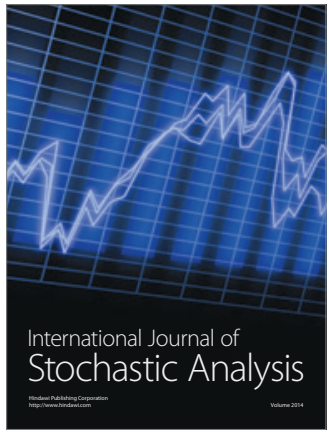

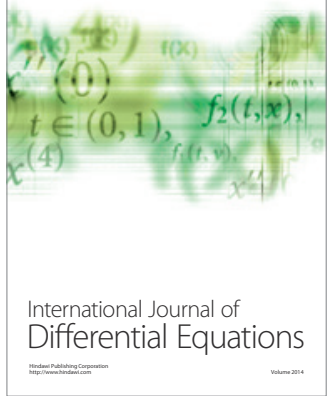
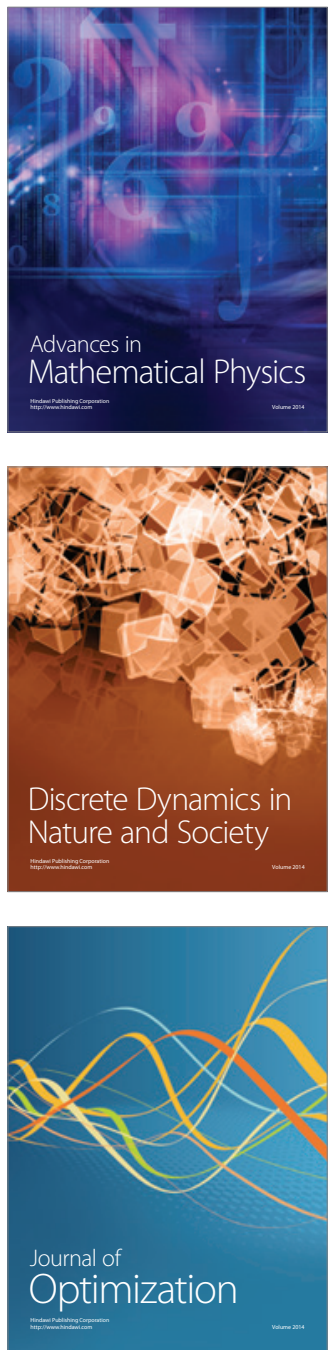